Generating Random Graphs Efficiently

\author{
Rajeev Raman
}

Technical Report 369

January 1991

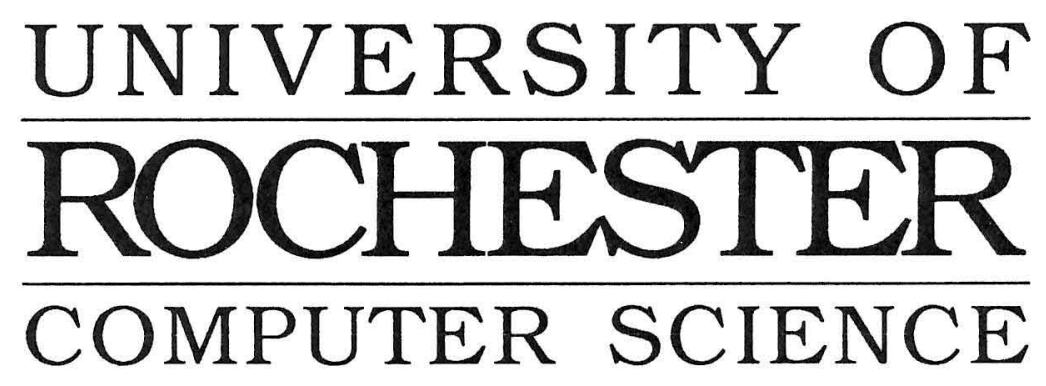




\title{
Generating Random Graphs Efficiently
}

\author{
Rajeev Raman \\ The University of Rochester \\ Computer Science Department \\ Rochester, New York 14627
}

Technical Report 369

January 1991

\begin{abstract}
We consider the algorithmic complexity of generating labeled (directed and undirected) graphs under various distributions. We describe three natural optimality criteria for graph generating algorithms, and show algorithms that are optimal for many distributions.
\end{abstract}

The University of Rochester Computer Science Department supported this work. 


\section{Introduction}

Many algorithms of practical interest turn out not to be amenable to precise analysis from a theoretical viewpoint. Typically these algorithms are heuristics that quickly give approximate solutions to problems that are difficult to solve exactly, like NP-complete problems. In such cases, the domain is either too complex to be analyzed formally, such as the KernighanLin algorithm for the Euclidean TSP [7] or simulated annealing [11], or the performance guaranteed by the best analysis is a severe underestimation of the observed performance of the algorithm in practice, as in the greedy MIS algorithm. In both these situations, an empirical analysis of the performance of the algorithm is needed. Other uses of such analyses may include gaining some idea of how an algorithm may perform, prior to analysing it theoretically. Since the output of the program may vary considerably with the input, the usual method is to run the algorithm on a large number of randomly generated test inputs, and to use statistical measures of the results obtained to judge performance. The importance of random graph generation in such a context is clear. Often the algorithm to be studied runs in time linear in the size of the graph, and almost always its run time is a polynomial of very small degree. It is clearly desirable that the time taken to construct an input to test the graph on should not exceed the time taken to actually run the test, and so the efficient generation of random graphs takes on significance.

Another fruitful area of application of the generation of random graphs arises in pure graph theory. Graph theorists sometimes attempt to catalog all the graphs of a given size and type. Such catalogs are used, among other uses, to settle conjectures by exhaustive enumeration or to come up with plausible conjectures. Such techniques are at their most useful in the study of random graphs where they have been used to obtain hints as to which way conjectures might be settled [16]. Again, due to the large number of graphs that need to be generated, the need for efficient generation is acute.

In this paper, we study ways of optimally generating random graphs with various properties. The notion of the optimality of a graph generating algorithm needs to be carefully defined: we have defined three natural ways in which such an algorithm may be considered optimal. To the best of our knowledge, this is the first such study of the algorithmic complexity of graph generation: all previous methods have either disregarded the complexity or have been content with polynomial-time generation (see [19] for a survey). We also consider some well-known types of random graphs, and exhibit efficient algorithms for these.

\section{Preliminaries}

\subsection{Definitions and Notation}

The order of a graph $G=(V, E)$ is the number of vertices in $\mathrm{G}$, and is usually denoted by $n$. The size of a graph is the number of edges it has, and is usually denoted by $m$. In an undirected graph, the degree of a vertex is the number of edges incident upon it. In a directed graph, the in-(out-) degree of a vertex is defined to be the number of edges entering (leaving) the vertex. A r-regular graph is an undirected graph each of whose vertices has degree equal to $r$. 
The probability of an event $A$ is denoted by $\operatorname{Pr}[A]$. If $X$ is a discrete random variable with distribution $D$ then we abbreviate $\operatorname{Pr}[X=i]$ by $D[i]$, omitting mentioning the random variable. The expected value (average) of a discrete random variable $X$ is denoted by $E(X)$. The uniform discrete distribution on the integers in the range $\{0, \ldots, k-1\}$ is denoted by $D_{k}$. The binomial distribution with parameters $z, p$ is denoted by $B(z, p)$, where

$$
B(z, p)[i]=\left\{\begin{array}{cc}
\left(\begin{array}{c}
z \\
i
\end{array}\right) p^{i}(1-p)^{z-i} & 0 \leq i \leq z \\
0 & \text { otherwise }
\end{array}\right.
$$

We abbreviate "let $x$ be the a value obtained by sampling distribution $D$ " by " $x \sim D$ ". Logarithms are to the base 2 unless otherwise indicated.

Our model of computation based on the uniform cost with logarithmic word size RAM $[1,6]$. The machine receives as input $n$, and after running a time polynomial in $n$ outputs a graph of order $n$ with the desired probability. We will assume that the machine can perform simple addressing and arithmetic operations on numbers with $O(\log n)$ bits each in constant time. We will assume that sampling once from $D_{k}$ takes constant time, provided $k$ is polynomial in $n$ (i.e., we are assuming the existence of a random integer generator). Having only a source of single random bits, it would be impossible to have a terminating algorithm that generated anything with probability not of the form $i / 2^{k}$ for some integer $k[10,14]$. We use the standard abbreviations for "uniformly at random" (u.a.r.) and "independent identically distributed" (i.i.d.).

\subsection{Optimality Criteria}

The operation of any algorithm $A$ on input $n$ can be represented by a tree, the leaves of which correspond to graphs. The branching factor of this tree will be finite and bounded by some polynomial in $n$. Let $U_{n}$ be the set of graphs of order $n$ that have a non-zero probability of being generated, and let $U=\cup_{i=0}^{\infty} U_{i}$. Denote the specified distribution on $U_{n}$ by $D_{n}$. For any graph $G$, let $d_{A}(G)$ the random variable that represents the depth of a leaf labeled with $G$. For any random variable $x$ let $\max \{x\}$ be the maximum value attained by it. Let $|G|=m+n$ be the size of the output. We have three notions of optimality:

\section{Definition 1 1. An algorithm $A$ is called optimal if}

$$
(\forall G \in U)\left[\max \left\{d_{A}(G)\right\} \text { is } O(|G|)\right] \text {. }
$$

2. An algorithm A is called PA-optimal (pointwise optimal on the average) if

$$
(\forall G \in U)\left[E\left(d_{A}(G)\right) \text { is } O(|G|)\right] \text {. }
$$

3. Let $G$ be a random variable that is distributed according to $D_{n}$. An algorithm $A$ is called A-optimal (optimal on the average) if

$$
\left(\forall G \in U_{n}\right)\left[E\left(d_{A}(G)\right) \text { is } O(E(|G|))\right] \text {. }
$$


For optimality, the worst-case time taken by the algorithm to generate the graph should be asymptotically the same as the size of the output, while for PA-optimality, the expected time taken to generate any graph is asymptotically the same as its output size. However, all an A-optimal algorithm has to guarantee is that for each $n$, the expected running time of the algorithm on input $n$ be asymptotically the same as the expected size of the output. Clearly, by definition, an algorithm is PA- and A-optimal if it is optimal, and is A-optimal if it is PA-optimal.

\section{Procedures Used}

\subsection{Sampling the Binomial Distribution}

The number of successes among $z$ i.i.d. Bernoulli variables each with probability $p$ of success is $B(z, p)$-distributed. This gives a simple method of generating such variables if $p$ is rational. Let $p=r / s$. Then we sample $D_{s} z$ times independently and return the number of times a number $<r$ was generated (this works within our model if $s$ is small enough). A faster approach is given by the generator of Ahrens and Dieter [2] which generates these variates in $O(\log z)$ time. For more references we direct the reader to [13, 12].

\subsection{Choosing Random Subsets}

The procedure below (from [20]) chooses a random $k$-subset of the integers from 0 to $n-1$ in $O(k)$ time using $O(n)$ space. It assumes an array $A[0 . . n-1]$ that is initialized such that location $A[i]$ contains the value $i$. At the end, the locations $A[n-k], \ldots, A[n-1]$ contain the desired random subset.

1. $i \leftarrow n$

2. $x \sim D_{i}$; swap $A[i]$ and $A[x]$;

3. $i \leftarrow i-1$; if $i>n-k$ go to 2 .

It is well known that any sequential RAM with an uninitialized memory can simulate one with an initialized memory with a constant factor increase in space and time, provided the initial value of each memory location in the initialized memory is computable in constant time from its address[1]. In our case, $A[j]$ would have contained the value $j$ had it been initialized, so this trick can be used. Also, with this method, re-initializing the array takes $O(1)$ time, so we have:

Lemma 2 Using $O(n)$ space, $m \geq 1$ random $k$ subsets of the integers $\{0, \ldots, n-1\}$ can be chosen in $O(k)$ time each.

By setting $k=n$ in the above algorithm and considering $A$ as an ordered vector we obtain a random permutation of the integers $\{0, \ldots, n-1\}$, so:

Corollary 3 Generating a random permutation of the integers $\{0, \ldots, n-1\}$ takes $O(n)$ time. 
The space requirement can be restricted to $O(k)$ if we are willing to settle for an expected $O(k)$ running time. The approach taken in [15] achieves this bound, if $k \leq n / 2$ (if not, the previous method is optimal). The idea is to keep generating random numbers in the range $\{0, \ldots, n-1\}$ until $k$ distinct numbers are obtained. If $k$ is as above, the expected number of trials required is $O(k)$. By using a hash table to store the partially generated subset, it is possible to check in $O(1)$ expected time whether or not a newly generated number is already present in the subset (note that since the set we store in the hash table is a subset of $\{0, \ldots, n-1\}$ that is generated u.a.r., the hash function $h(x)=x \bmod s$ where $s$ is the size of the hash table would have this performance).

Lemma 4 Choosing a random $k$ subset of the integers $\{0, \ldots, n-1\}$ takes expected $O(k)$ time using $O(k)$ space.

\subsection{Computing "Pairing" Functions}

Often, we will have to select an edge at random from a set of edges. For this purpose, it is extremely useful to have a bijection between all possible edges of a graph on the vertex set $\{0, \ldots, n-1\}$ and the integers in the range 0 through $\left(\begin{array}{c}n \\ 2\end{array}\right)-1$. Such mappings appear as "pairing" functions in the literature, but the complexity of computing these mappings is largely ignored. We present a bijection that can be computed in constant time on our model of computation (which corresponds roughly to using only integer operations in actual computers). The computation requires precomputed tables of size $O(n)$.

Lemma 5 There is a mapping that can be computed in $O(1)$ time on our model of computation.

Proof. We use the following mapping:

$$
\begin{aligned}
\operatorname{pair}(s, t) & =(n-1-s+t)(n-s+t) / 2+(n-1-s) \\
\operatorname{unpair}_{s}(z) & =n-1-(z-(j)(j+1) / 2) \\
\operatorname{unpair}_{t}(z) & =j-(z-(j)(j+1) / 2), \text { where } \\
j & =\lfloor(\sqrt{1+8 z}-1) / 2\rfloor
\end{aligned}
$$

Note that in our model directly computing $f(z)=\lfloor(\sqrt{1+8 z}-1) / 2\rfloor$ is not permitted. We observe first that $y=\lfloor(\sqrt{1+8 z}-1) / 2\rfloor$ implies that:

$$
(\exists \delta)[0 \leq \delta<1 \wedge z=(y+\delta)(y+\delta+1) / 2]
$$

So, for a given $y$, the minimum integral value of $z$ such that $f(z)=y$ is $y(y+1) / 2$ and the largest such $z$ is $(y+1)(y+2) / 2-1$. For $1 \leq z \leq n$ we compute $f(z)$ directly using the above fact, and store the resulting values in a table.

For larger values of $z$, the above approach fails, since the table would be too large. We will directly compute the function $\sqrt{x}$ to within an additive $O(1)$ factor. After this, we will compute $f(z)$ to within $O(1)$ using the approximate value of $\sqrt{1+8 z}$ and then use linear search to find the correct value of $f(z)$. To compute $\sqrt{x}$ to within an $O(1)$ additive factor, 
we use the following precomputed tables: a table with $\lfloor\log x\rfloor \operatorname{computed}$ for $1 \leq x \leq n$ and a table with $\lfloor\sqrt{x}\rfloor$ computed in double precision for $1 \leq x \leq n$. By double precision we mean that we store, for each $x$, numbers $k_{1}$ and $k_{2}$ such that $\left\lfloor\left(k_{1}+k_{2} / n\right)^{2}\right\rfloor=x$.

Using the tables for the log function, we first compute an approximate value for $\sqrt{x}$ as $y=2^{\lfloor(\lfloor\log (\lceil x / n\rceil)\rfloor+\lfloor\log n\rfloor) / 2\rfloor}$. Now we compute $y^{\prime}=\sqrt{\lfloor x / y\rfloor} \sqrt{y}$ using values from the double precision table that we precomputed. It is easy to show that the value $y^{\prime}$ is within $O(1)$ of the actual value of $\sqrt{y}$. The essential point is that $\sqrt{\lfloor x / y\rfloor}$ is within $O\left(x^{-1 / 2}\right)$ of $\sqrt{x / y}$.

We would note that our mapping is perhaps a little complex to implement in practice, and for most applications the mapping $\operatorname{pair}(s, t)=(s-1) n+t$ would be preferable. This mapping is not injective, and so we may be forced to reject some randomly integers since they would not correspond to legal edges. This would be a relatively infrequent occurrence, however.

\section{Generation of Random Graphs}

\subsection{Graphs with Specified Number of Edges}

The distribution $E N(n, m)$ (Edge Number) is such that all (undirected) graphs with order $n$ and size $m$ are equally likely to be generated. With the mapping of lemma 5 , the problem reduces to selecting a random subset of size $m$ of the set of integers 0 through $\left(\begin{array}{l}n \\ 2\end{array}\right)-1$. By lemma 2 and lemma 4 this can be done in expected $O(m)$ time using $O(m)$ space, or in $O(m)$ time using $O\left(n^{2}\right)$ space.

We have thus shown:

Theorem 6 The distribution $E N(n, m)$ can be optimally generated using $O\left(n^{2}\right)$ space and $P A$-optimally generated using $O(m+n)$ space.

\subsection{Specified Edge Probability Graphs}

The distribution $\operatorname{EP}(n, p)$ (Edge Probability) is parametrized by a probability $p \in[0.0,1.0]$ and the order $n$ of the graph. Labeled undirected graphs of order $n$ are generated in such a way that the probability of each possible edge being present is $p$ independently of the other edges. The naive method consists of considering every possible edge in turn and adding it to the graph independently with probability $p$. This method takes $O\left(n^{2}\right)$ time, but as the expected number of edges is $p m$, it is non-optimal when $p$ varies with $n$ such that $p(n)$ is $o(1)$. Also, even when $p$ is a constant, this algorithm is only A-optimal.

A better method depends on the fact that the number of edges in a $E P(n, p)$-distributed graph, $k$, is binomially distributed with parameters $m$ and $p$. We mention that Tinhofer had a similar, if somewhat less efficient, variant of this idea [18]. This procedure consists of two stages. In the first, we sample the $B(m, p)$ distribution to obtain a value $k$ for the number of edges in the graph. Using the efficient procedures mentioned above, we can do this in time $O(\log m)=O(\log n)$. In the second stage, we randomly generate a graph of order $n$ and size $k$ such that all graphs of order $n$ and size $k$ are equally likely to have been 
generated. From well-known properties of the binomial distribution it is easily seen that in any graph generated by the above procedure, the probability that any particular edge is present is $p$, independently of all other edges. From theorem 6 we can bound the (expected or deterministic) time complexity of the second phase by $O(m+n)$. Thus:

Theorem 7 The distribution $E P(n, p)$ can be optimally generated using $O\left(n^{2}\right)$ space or $P A$-optimally using $O(m+n)$ space.

Let $C(n), E(n)$ and $E u(n)=C(n) \cap E(n)$ be the uniform distributions over all labeled graphs with $n$ vertices that are connected, have all vertex degrees even, and are Eulerian, respectively. Noting that $E P\left(n, \frac{1}{2}\right)$ specifies that all labeled graphs on $n$ vertices are equally likely, we obtain as a corollary:

Corollary $8 C(n)$ and $E u(n)$ can be PA-optimally generated with $O(m+n)$ space, and $E(n)$ can be optimally generated with $O\left(n^{2}\right)$ space and PA-optimally with $O(m+n)$ space.

Proof. We generate $C(n)$ using a restarting procedure, i.e., by generating graphs from $E P\left(n, \frac{1}{2}\right)$ and testing to see if they are connected (such an approach was mentioned by Tinhofer). We note that the probability of generating an unconnected graph is less than $2^{-\Omega(n)}$. This is because the number of connected graphs of order $n, c_{n}$, obeys the following recurrence $([8])$ :

$$
c_{n}=2^{\left(\begin{array}{c}
n \\
2
\end{array}\right)}-\frac{1}{n} \sum_{k=1}^{n-1} k\left(\begin{array}{l}
n \\
k
\end{array}\right) 2^{\left(\begin{array}{c}
n-k \\
2
\end{array}\right)} c_{k}
$$

Observing that $c_{k}<2\left(\begin{array}{l}k \\ 2\end{array}\right)$ and that $\left(\begin{array}{c}n \\ 2\end{array}\right)=\left(\begin{array}{c}n-k \\ 2\end{array}\right)+\left(\begin{array}{c}k \\ 2\end{array}\right)+k(n-k)$, it is easy to show that the latter term is asymptotically smaller by a factor of at least $2^{-n / 2}$. Thus the expected cost to generate a graph $G$ of size $m$ is $T \leq 2^{-n / 2}\left(O\left(n^{2}\right)+T\right)+\left(1-2^{-n / 2}\right) O(m+n)$, from which we obtain the PA-optimality of this restarting procedure.

The procedure for generating $E(n)$ depends on the 1-1 correspondence between all even degree graphs on $n$ vertices and all graphs with $n-1$ vertices [8]: with each graph $G$ on $n-1$ vertices associate the even-degree graph obtained by adding a new vertex $v_{n}$ and joining all odd-degree vertices of $G$ to $v_{n}$. The corollary follows.

For $E u(n)$ we use a restarting procedure again. We generate a member of $E(n)$ as above and check to see if it is connected. Let $G$ be a random element of $E(n)$ generated as above and let $G^{\prime}=G-\left\{v_{n}\right\}$ be the graph generated from $\operatorname{EP}\left(n-1, \frac{1}{2}\right)$ in the above procedure. $G$ will be unconnected only if either $G^{\prime}$ is unconnected or $G^{\prime}$ is an even degree graph, in which case $v_{n}$ will be an isolated vertex. The probability that $G^{\prime}$ is not connected is at most $2^{-\Omega(n)}$, as argued above. The probability that $G^{\prime}$ is an even degree graph is exactly the fraction of all $n-1$ vertex labeled graphs that are even degree. From the 1-1 correspondence between between all even degree graphs on $n-1$ vertices and all graphs with $n-2$ vertices, we deduce that this probability is at most $\frac{2\left(\begin{array}{c}n-2 \\ 2\end{array}\right)}{2\left(\begin{array}{c}n-1 \\ 2\end{array}\right)}$ which is also of the order of $2^{-\Omega(n)}$. We thus observe that the restarting procedure has a probability $2^{-\Omega(n)}$ of failing on each iteration, giving us a PA-optimal algorithm for $E u(n)$, by applying the above reasoning. $\square$ 
A similar approach can be taken towards generating labeled directed graphs with a specified number of edges.

\subsection{Digraphs with Specified Out-degree}

The distribution $D O(n, k)$ (Directed, Out-degree) specifies that all order $n$ digraphs where each vertex has outdegree $k$ are equally likely. Such graphs can be obtained by generating, for each vertex, a set of its successors of size $k$. If self-loops are allowed, lemma 2 directly gives us a $O(k n)=O(m+n)$ algorithm. If not, a variant of the method of lemma 2 needs to be used. If while generating the neighbors of vertex $v \in\{0, \ldots, n-1\}$, we know a position $j$ such that $A[j]=v$, we can generate a random $k$-subset of $\{0, \ldots, n-1\}-v$ by swapping the contents of $A[j]$ and $A[n-1]$ before starting the selection. To find such a $j$ efficiently, we can maintain an 'inverse' array $B[0 \ldots n-1]$ which has the property that $B[i]=j$ iff $A[j]=i$, and update it each time $A$ is modified. Thus:

Theorem 9 Given $n$ and $k$, a graph distributed according to $D O(n, k)$ can be generated optimally.

\subsection{Graphs with Specified Degrees}

A sequence of integers $d=\left(d_{1}, d_{2}, \ldots, d_{n}\right), 1 \leq d_{i} \leq n-1$ is called a degree specification. A degree specification is called graphical if there is a graph on the vertices $\{1, \ldots, n\}$ such that for all $i, d(i)=d_{i}$. The distribution $D S(d)$ (Degree Specification) states that all graphs with degree specification $d$ are to be generated with equal probability (regular graphs form a special case of particular interest). Such graphs appear to be hard to generate u.a.r., and this problem, along with the related problem of exactly counting the numbers of such graphs, has remained open for several years. Tinhofer [18] gave a method for generating such graphs non-uniformly such that the a posteriori probability that the graph was generated can be obtained. His method runs in $O\left(n^{4}\right)$ time for simple generation and $O\left(n^{5}\right)$ time for generation with probabilities computed. Jerrum and Sinclair [9] describe a polynomialtime algorithm that generates such graphs with a distribution that can be made arbitrarily close to uniform, by increasing the running time appropriately. This algorithm only works for the set of P-stable degree specifications (which, however, includes all regular degree specifications as a special case) and is of doubtful practical utility since the running time to achieve any reasonable approximation is a polynomial of very high degree.

We present two practical algorithms that run quickly but do not guarantee uniform generation. By increasing the run time by a factor of $n$, the a posteriori probability that the graph was generated can be computed as well. While the first algorithm is optimal, the distribution that it generates may be undesirable since it produces with high probability graphs that are similar to a canonical graph. The second algorithm is a much more efficient implementation of Tinhofer's algorithm, and produces a distribution that may be better for some applications.

We now describe an optimal algorithm for generating a graph with degree specification $d$ if one exists in $O(m+n)$ time. Define a degree sequence to be a degree specification 
sorted into non-decreasing order. The following claim (see [3]) is proved again here for completeness:

Claim 10 Let $d=\left(d_{1}, \ldots, d_{n}\right)$ be a degree sequence. Then the degree sequence $d^{\prime}=\left(d_{2}-\right.$ $\left.1, \ldots, d_{d_{i}+1}-1, d_{d_{i}+2}, \ldots, d_{n}\right)$ is graphical iff $d$ is.

Proof. It is trivial to prove that if $d^{\prime}$ is graphical, then $d$ is, so we will prove the other direction. Suppose $d$ is graphical. Then there is a graph $G=(V, E)$ such that $G$ has degrees specified by $d$. Let $v$ be any vertex in $G$ such that $d(v)=d_{1}$. If $(v, w) \in E$ is an edge and $(v, x) \notin E$ and $d(w)<d(x)$, then there is a graph $G^{\prime}=\left(V, E^{\prime}\right)$ with the same degree sequence such that $(v, x) \in E^{\prime}$ is an edge and $(v, w) \notin E^{\prime}$ is not. To see this, note that since $d(w)<d(x)$, there is a vertex $y$ such that $(x, y) \in E$ but $(w, y) \notin E$. Setting $E^{\prime}=E-\{(v, w),(x, y)\} \cup\{(v, x),(w, y)\}$ we see that the above statement is true. Repeating this construction as often as necessary we come to a point where for any vertex $w$ that is a neighbor of $v$ and for any vertex $x$ that is not a neighbor of $v, d(w) \geq d(v)$. Deleting $v$ from this graph gives a graph with degree sequence $d^{\prime}$, which proves the claim.

Let the residual degree $r_{i}$ of a vertex $i$ is the number of edges it needs to bring its degree up to $d_{i}$. The residual degree sequence of a graph is formed by arranging the residual vertex degrees in non-increasing order. Using the above claim, we have the following method of generating a canonical graph with degree specification $d$ :

Repeatedly join the vertex $v$ with the largest residual degree, $k$, to the $k$ vertices that have the $k$ largest residual degrees.

Claim 11 The above algorithm can be implemented to run in $O(m+n)$ time.

Proof. Suppose we have to store a set $S$ variables $x_{1}, \ldots, x_{k}, x_{i} \in\{0, \ldots, n\}$ and perform the operations $\max (S)$, which returns the index of a variable of maximum value and $\operatorname{dec}(j, S)$, which decrements the value of $x_{j}$ by 1 if it is non-zero. In [5] it is shown how to implement these operations in $O(1)$ time each.

The solution involves maintaining a list of lists. In the top-level list we keep as list headers each number in the range $\{0, \ldots, n\}$ that is the value of some variable in $S$. We keep this list of list headers sorted. With each of these headers we associate a list of the indices of variables with that value. Since the value of a variable is decreased only by $O(1)$ in each step, finding the list into which a variable must be inserted can be done in $O(1)$ time using linear search.

Now, starting with the canonical graph $G=(V, E)$ obtained above, we apply perturbations to the graph in a manner similar to Jerrum and Sinclair. Let $m=\left(\sum_{i=1}^{n} d_{i}\right) / 2$ be the number of edges in the graph.

1. If the current graph $G$ has degree specification $d$ pick an edge $(i, j)$ at random and delete it from $E$.

2. Otherwise, $G$ has two vertices that currently have degree one less than they should. Pick one of these at random. Let this vertex be $i$. Pick an edge $(i, k) \notin E$ at random and add it to $E$. If now $k$ has degree one more than it should, pick an edge $(k, l) \in E$ at random and delete it from $E$. 
3. If $2 m$ iterations have not been completed, go to 1 .

4. If $G$ does not have degree specification $d$, find the $G^{\prime}$ closest to $G$ that has degree specification $d$ and output it.

Theorem 12 The above algorithm generates all graphs with degree specification d and runs in $O(m+n)$ time.

Proof. We will show that $2 m$ such alterations suffice to transform the canonical graph $\bar{G}$ into any graph $G$ with the required degree sequence. Note that $G^{\prime}=\bar{G} \oplus G$ is an even-degree graph with at most $2 m$ edges. $G^{\prime}$ can be broken up into disjoint Eulerian components. Consider an Euler tour on one of these components that alternates edges from $\bar{G}$ and $G$. It is clear that if the above perturbations convert $\bar{G}$ into a graph $G$ with degree specification $d$ then the algorithm must have performed an Euler tour on some Eulerian component of $\bar{G} \oplus G$. Since the number of edges in $\bar{G} \oplus G$ is at most $2 m, 2 m$ iterations of steps 1-3 suffice to convert $\bar{G}$ into any graph with specification $d \dot{I}$ n case the graph at the end of the process does not have degree specification $d$, it is a simple matter to "complete" the Euler tour the algorithm was in, to give a graph with degree specification $d$. It is also easy to make the random choices in steps 1 and 2 efficiently.

We now give an efficient implementation of an algorithm that is rather like Tinhofer's. We first permute the edges randomly. Then we consider each edge in turn according to this permutation, adding it if the resulting residual degree sequence is graphical. It can be shown [3] that the residual degree sequence is graphical iff, for $1 \leq k \leq n$,

$$
\sum_{i=1}^{k} r_{i} \leq k(k-1)+\sum_{i=k+1}^{n} \min \left\{k, r_{i}\right\} .
$$

Thus, we need the following operations on residual degree sequences: test( $e$ ), which returns true if the residual degree sequence $r^{\prime}$ obtained by adding the edge $e$ to the graph is graphical and false otherwise; and $a d d(e)$, which adds the edge $e$ to the graph and updates the residual degree sequence from $r$ to $r^{\prime}$. A naive implementation would result in an $O\left(n^{2}\right)$ complexity for test $(e)$ and an $O(1)$ complexity for $\operatorname{add}(e)$. Note that while test $(e)$ may be called $O\left(n^{2}\right)$ times, $\operatorname{add}(e)$ will be called only $O(m)$ times, so often we can decreasing the complexity of test $(e)$ at the expense of increasing the complexity of $\operatorname{add}(e)$ and still obtain an improved algorithm.

For any residual degree sequence $r=\left(r_{1}, \ldots, r_{n}\right)$, let $k_{0}(r)=\max \left\{i \mid r_{i}>i\right\}$. Let $r$ be the current residual degree sequence, and let $r$ be graphical (initially, $r=d$ ). Let $n_{k}$, $0 \leq k \leq n-1$, be the number of times the number $i$ appears in $r$. We let $N_{k}=\sum_{i=0}^{k} n_{i}$, for $0 \leq k \leq n-1$ and $I_{k}=r_{k}+N_{k-1}-n+1$, for $1 \leq k \leq n$. We now note that equation 1 may be restated as:

$$
\text { for } 1 \leq k \leq n, S_{k}=\sum_{i=1}^{k} I_{i} \leq 0 .
$$

Equation 2 need be checked only for $1 \leq k \leq K=k_{0}(r)$. This is because $I_{k}<0$ for all $k>K$. We will show that the operations $\operatorname{dec}(i)$, inc(i) and test( $)$ can be performed 
in $O(\log K)$ time, where $\operatorname{dec}(i)$ and $i n c(i)$ decrement or increment $r_{i}$ and test () tests if the current residual degree sequence is graphical or not. Clearly $\operatorname{add}(e)$ and test $(e)$ above can be implemented using these primitives.

The effect of decreasing $r_{i}$ from $x$ to $x-1$ is to decrease $I_{i}$ by 1 , decrease $I_{x+1}$ by 1 and increase $I_{x}$ by 1 . We keep the sums $S_{1}, S_{2}, \ldots, S_{K}$ at the leaves of a binary tree $T$ with depth $\log K$ in the order above. We perform the following operations on these sums: change $(j, \delta)$ which sets $S_{i}$ to $S_{i}+\delta$ for all $j \leq i \leq K$ and $\max \left(\right.$ ), which returns $\max \left\{S_{1}, \ldots, S_{K}\right\}$. We can perform each of these operations in $O(\log K)$ time, by storing at each internal node $v$ of the tree the value of the maximum value $M$ in the subtree rooted at $v$, as well as a quantity $\Delta$ that represents the sum of all values $\delta$ such that some previous operation change $(i, \delta)$ changed all values in the subtree rooted at $v$ but did not change all values in the subtree rooted at $v$ 's parent. (I.e, the current value of $S_{i}$ is obtained by adding to the value stored at the $i$ th leaf the $\Delta$ values at all the ancestors of the $i$ th leaf.) To implement change $(j, \delta)$, instead of directly changing the values $S_{j}, \ldots, S_{K}$, we split these up into at most $O(\log K)$ canonical subsets, each of which corresponds to a complete subtree of $T$, and add $\delta$ to the $\Delta$ values stored at the roots of these subtrees. This may cause $O(\log K)$ maximum values to change, but these values will be at children of nodes that are on the path from $j$ to the root, and so percolating these values up can be done in $O(\log K)$ time.

These improvements give an algorithm runs in time $O\left(n^{2} \log K\right)$ where $K=k_{0}(d)(K=t$ for $t$-regular graphs):

Theorem 13 Tinhofer's algorithm can be implemented to run in time $O\left(n^{2} \log K\right)$ where $K=k_{0}(d)$.

Remark In this algorithm as stated, the expected number of edges examined before the algorithm terminates is $\Theta\left(n^{2}\right)$, giving an expected running time of $\Theta\left(n^{2} \log K\right)$. This is because if $x$ edges are examined by the algorithm before the $m-1$ st edge is chosen for inclusion, the unique edge that will satisfy the regularity test will be discovered after examining $\frac{1}{2}\left(\left(\begin{array}{c}n \\ 2\end{array}\right)-1+x\right)$ more edges on the average, for a total of $\Theta\left(n^{2}\right)$. This algorithm is thus not even within a $O(\log K)$ factor of A-optimality. To come closer to A-optimality, we suggest the following approach: we use the above algorithm until about $m / n$ edges have been chosen. Suppose the residual degree specification is at this time is $r^{\prime}$. We now make a recursive call to generate a graph with degree specification $r^{\prime}$. Since it takes $O(n)$ time to initialize the data structures for a recursive call, the extra time taken would be $O(m)$. We conjecture that on the average, selecting $O(m / n)$ edges could be done by examining only $O(m / n)$ edges.

\subsection{Labeled Trees}

The distribution $\operatorname{LT}(n)$ states that all free (unrooted) labeled trees of order $n$ are equally probable. Cayley's formula states that there are $n^{n-2}$ such trees. Prüfer proved an easily computable 1-1 correspondence between labeled trees of order $n$ and sequences of integers between 1 and $n$ of length $n-2$. This suggests the following approach, used in [15]: generate a random sequence of $n-2$ integers between 1 and $n$, and output the corresponding tree. This is the basis of both the algorithm given in [15] and our algorithm. The algorithm in 
[15] runs in time $O\left(n^{2}\right)$, though it can easily be made to run in time $O(n \log n)$. We describe an implementation that takes linear time.

Prüfer's method of obtaining a unique labeled tree with $n$ vertices labeled $\{1, \ldots, n\}$ from a sequence $P$ of $n-2$ numbers each in the range $\{1, \ldots, n\}$ is as follows (here we assume $n \geq 3$ ) :

1. Let $E$ be the set of all the integers from 1 to $n$ that do not appear in $P$.

2. Let $k$ be the smallest integer in $E$, and $m$ be the the first element in $P$. Add the edge $(k, m)$ to the tree. Delete $m$ from the front of $P$, and $k$ from $E$. If $m$ does not appear elsewhere in the sequence, add $m$ to $E$.

3. If $P$ is now empty, there must be exactly 2 elements left in $E$. Call these $n$ and $o$. Add $(n, o)$ to the tree and quit.

In [15] the set $E$ is represented by an unsorted array, and this implementation causes the running time to be $O\left(n^{2}\right)$. Using a heap instead would get the running time down to $O(n \log n)$. We can get a quicker algorithm, however. The observation is that in linear time, we can determine the operations that would be performed on the set $E$ by this algorithm, which would be an intermixed sequence of commands of the form insert $(x)$ or deletemin (the latter operation means to report the current minimum element in $E$ and to delete it). This is because in $O(n)$ time we can determine the elements initially placed in $E$, as well as the last position any label occurs in the sequence (which is the point it is inserted into $E$ ), and this determines the position of the insert operations with respect to the deletemin operations. The problem of determining the answers to a sequence of such operations, when the operations are presented all at once, is the well-studied off-line min problem. In [1, 17] a simple algorithm is presented for this problem that takes time $O(n \alpha(n))$ to answer a sequence of $n$ inserts and deletemins. (Here $\alpha(n)$ is a very slowly growing function such that $\alpha(n) \leq 3$ for all possible practical values of $n$.) Alternatively the more complex method of [6] achieves a true linear running time for solving this problem.

1. Generate the sequence $P=\left\{p_{i}\right\}_{i=1}^{n-2}$.

2. In linear time obtain $E 0$, the set of all elements in $\{1, \ldots, n\}$ that do not appear in $P$, as well as the sequence $L=\left\{l_{i}\right\}_{i=1}^{n-2}$, where $l_{i}=1$ if the value $p_{i}$ does not reappear at some later point in $P$, and 0 otherwise.

3. Construct the sequence of operations $O$ in the following way: for each $x \in E 0$, put an operation $\operatorname{insert}(x)$ before the first deletemin operation. Thereafter, for $1 \leq i \leq n-2$, do the following: append the $i$ th deletemin to the sequence, followed by the operation insert $\left(p_{i}\right)$ if $l_{i}=1$. Finally append two deletemin commands to $O$.

4. Solve the off-line min problem with operation sequence $O$.

5. Let $a_{i}$ be the answer to the $i$ th deletemin operation. Output the following edges: $\left(a_{1}, p_{1}\right), \ldots,\left(a_{n-2}, p_{n-2}\right),\left(a_{n-1}, a_{n}\right)$.

Theorem 14 The distribution $L T(n)$ can be optimally generated. 


\subsection{Geometric Graphs}

The parameters here are $n$, the order of the graph, and three real numbers $X, Y$ and $r$. The distribution $G G(n, X, Y, r)$ is obtained as follows: drop $n$ points at random in the rectangle $\{(x, y) \mid 0 \leq x<X, 0 \leq y<Y\}$ and join any pair of points that are less than $r$ apart by an edge. This distribution was introduced in [11] as a natural distribution for examples to test heuristics for NP-complete problems like the TSP (which satisfy the triangle inequality) on. The straightforward method results in an $O\left(n^{2}\right)$ complexity independent of $X, Y$ and $r$.

The geometric nature of this problem suggests techniques from computational geometry. Using the "circle" searching algorithm of [4] we can get a $O(n \log n+m)$ algorithm, which would be A-optimal whenever the values of $X, Y$ and $r$ are such that $E(|G|)=O(n \log n)$. We now describe a considerably simpler algorithm that is A-optimal for all values of $X, Y$ and $r$.

We divide the $X \times Y$ rectangle into $m=\lceil X / r\rceil \cdot\lceil Y / r\rceil$ subrectangles with longer side at most $r$ such that $R_{i, j}=\{(x, y) \mid i r \leq x<\min \{(i+1) r, X\}, j r \leq y<\min \{(j+1) r, Y\}\}$, for $0 \leq i \leq\lceil X / r\rceil-1,0 \leq j \leq\lceil Y / r\rceil-1$. We assign these rectangles numbers systematically, e.g., \# $(i, j)=j\lceil X / r\rceil+i$. Now we generate the $n$ points, and for each point, determine the rectangle $R_{i, j}$ in which it lies. For each rectangle $R_{i, j}$ that already has has at least one point located inside it, we store \# $(i, j)$ in a hash table with $2 n$ buckets using the hash function $f(k)=k \bmod 2 n$, as well as a record with a list of points inside that square. The expected time to search in the hash table is clearly $O(1)$.

Now we consider the points one by one: for each point $x$, we obtain a list $L_{x}$ of all the points in its rectangle as well as in its adjacent rectangles, by looking up the numbers of the adjacent squares in the hash table. Now in $O\left(\left|L_{x}\right|\right)$ time we determine the set $A_{x}$ of all the points in $L_{x}$ that are less than $r$ away from $x$. The running time of this algorithm is:

$$
T \leq c \sum_{x \in V}\left|L_{x}\right|+1
$$

Since $E\left(\left|L_{x}\right|\right)=9 r^{2} n / X Y$, we have by linearity of expectation,

$$
E(T) \leq c \sum_{x \in V} E\left(\left|L_{x}\right|\right)+1 \leq c\left(n+9 r^{2} n^{2} / X Y\right) .
$$

However, since the expected size of the graph generated is $O\left(n+r^{2} n^{2} / X Y\right)$, we have:

Theorem 15 The above algorithm generates $G G(n, X, Y, r)$ A-optimally.

We conjecture that the above algorithm could be modified to achieve PA-optimality.

\section{Open Problems and Directions for Future Research}

We now list some open problems:

1. Find a way (in our model) of choosing a random $k$-subset in $O(k)$ time with $O(k)$ space. This would imply the optimality of the algorithms of theorems 6 and 7 even using linear space. 
2. Find an optimal uniform generator for $D S(d)$, or at least a practical one. More concretely, is the modified algorithm of theorem 13 within a log factor of optimality?

3. Determine whether or not the algorithm of theorem 15 is PA-optimal.

4. Find optimal algorithms for unlabeled graphs and for other kinds of labeled graphs. 


\section{References}

[1] A. V. Aho, J. E. Hopcroft, and J. D. Ullman. The Design and Analysis of Computer Algorithms. Addison-Wesley, Reading, Massachussetts, USA, 1974.

[2] J. A. Ahrens and U. Dieter. Computer methods for sampling from gamma, beta, poisson and binomial distributions. Computing, 12:223-246, 1974.

[3] J. G. Bondy and U. S. R. Murty. Graph Theory and Applications. North-Holland, 1976.

[4] B. Chazelle and H. Edelsbrunner. Optimal solutions to a class of point retreival problems. In Proc. 12th International Colloquium on Automata, Lanugages and Program$\operatorname{ming}(I C A L P), 1985$.

[5] P. Dietz and D. Sleator. Two algorithms for maintaining order in a list. In Proc. 19th ACM STOC, pages 365-372, 1987.

[6] H. N. Gabow and R. E. Tarjan. A linear-time algorithm for a special case of disjoint set union. Journal of Computer and System Sciences, 30:209-221, 1985.

[7] M. R. Garey and D. S. Johnson. Computers and Intractability. W. H. Freeman and Company, 1979.

[8] F. Harary and E. M. Palmer. Graphical Enumeration. Academic Press, 1973.

[9] M. R. Jerrum and A. J. Sinclair. Fast uniform generation of regular graphs. Technical Report CSR 281-88, Department of Computer Science, University of Edinburgh, October 1988. To appear in Theoretical Computer Science.

[10] M. R. Jerrum, L. G. Valiant, and V. V. Vazirani. Random generation of combinatorial structures from a uniform distribution. Theoretical Computer Science, 48:169-188, 1986.

[11] D. S. Johnson, C. R. Aragon, L. A. McGeoch, and C. Schevon. Optimization by simulated annealing: an experimental evaluation (part I). Technical report, AT\&T Bell Labs, 1987.

[12] V. Kachitvichyanukul and B. Schmeiser. Binomial random variate generation. Communications of the ACM, 31(2):216-222, February 1988.

[13] D. E. Knuth. The Art of Computer Programming, Vol. 2: Seminumerical Algorithms. Addison-Wesley, Reading, Massachussetts, USA, 1973.

[14] D. E. Knuth and A. C. Yao. The complexity of non-uniform random number generation. In J. F. Traub, editor, Algorithms and Complexity: New Directions and Recent Results. Academic Press, 1976.

[15] A. Ninjenhuis and H. S. Wilf. Combinatorial Algorithms. Academic Press, 1975. 
[16] E. M. Palmer. Graphical Evolution: An Introduction to the Theory of Random Graphs. John Wiley, 1985.

[17] R. E. Tarjan. Efficiency of a good but not linear set union algorithm. Journal of the ACM, 1975.

[18] G. Tinhofer. On the generation of random graphs with given properties and known distributions. Applied Computer Science (Munich), 13:265-297, 1979.

[19] G. Tinhofer. Generating graphs uniformly at random. Computing, Supplementum, $7: 235-255,1990$.

[20] N. C. Wormald. Generating random regular graphs. Journal of Algorithms, 5:247-280, 1984. 